\title{
Translational health economics: The key to accountable adoption of in vitro diagnostic technologies
}

\section{Christopher P Price $\stackrel{1}{1}$}

Jane Wolstenholme $\underline{1}$

\section{Patrick McGinley $\underline{2}$}

\section{Andrew St John $\underline{3}$}

1., University of Oxford, Oxford, UKAQ1

${ }^{2}$ Maidstone and Tunbridge Well NHS Trust, Maidstone Hospital, Maidstone, UK

${ }^{3}$ ARC Consulting, Perth, Australia

Corresponding author:

Christopher P Price, University of Oxford, Primary Care Building, Radcliffe Observatory Quarter, Oxford OX2 6GG, UK. Email: cpprice1@gmail.com

Adoption of new technologies, including diagnostic tests, is often considered not to deliver the expected return on investment. The 
reasons for this poor link between expectation and outcome include lack of evidence, variation in use of the technology, and an inability of the health system to manage the balance between investment and disinvestment associated with the change in care pathway. The challenges lie in the complex nature of healthcare provision where the investment is likely to be made in the jurisdiction of one stakeholder while the benefits (as well as disbenefits) accrue to the other stakeholders. A prime example is found in the field of laboratory medicine and the use of diagnostic tests. The current economic tools employed in healthcare are primarily used to make policy and strategic decisions, particularly across health systems, and in purchaser and provider domains. These tools primarily involve cost effectiveness and budget impact analyses, both of 
which have been applied in health technology assessment of diagnostic technologies. However, they lack the granularity to translate findings down to the financial management and operational decision making at the provider department level. We propose an approach to translational health economics based on information derived from service line management and time-driven activitybased costing, identifying the resource utilisation for each of the units involved in the delivery of a care pathway, before and after adoption of new technology. This will inform investment and disinvestment decisions, along with identifying where the benefits, and disbenefits, can be achieved for all stakeholders.

Keywords: financial management, health economics, laboratory medicine, technology adoption, translational leadership, value-based healthcare 


\section{Introduction}

In recent years, concerns have been expressed regarding both the clinical and cost effectiveness of technologies employed in the provision of healthcare. 1 This, together with the perception of a poor return on investment for diagnostic technologies has to be considered in the context of calls to improve the quality of care and improve outcomes,,$\underline{2}$ and reduce costs. $\frac{3}{A}$ range of solutions have been proposed to meet these objectives including adopting a more integrated approach to care, with a more outcomes-, and value-based,,$\underline{4}$ approach to evaluating healthcare interventions. Amongst providers, there has been a greater focus on improving efficiency $\underline{5}$ and productivity, $\underline{6}$ while also reducing waste. $\underline{\underline{7}}$

Barriers to the adoption of new technology in healthcare have been recognised, including (i) lack of clarity in the unmet need, (ii) lack of evidence of effectiveness, (iii) reimbursement not linked to impact on outcomes, (iv) silo budgets, (v) failure to disinvest in redundant resources, $\underline{\text { - }}$ $\underline{11}$ as well as cultural, human behavioural and organisational issues associated with change. Furthermore, the complexity of the activities associated with the delivery of healthcare is also recognised as a challenge to demonstrating clinical and cost effectiveness, as well as a transition to value -and outcome-based care. $\underline{12}$

While these observations can be attributed to many technologies employed in the delivery of healthcare, there are some unique aspects demonstrated in the sphere of in vitro diagnostic tests including the following: 
1. The effectiveness of any test requires not only that the result is delivered to set standards (e.g. accuracy, precision, timeliness and relevance) but that the result is acted upon appropriately (and again, in a timely fashion).

2. The speed with which the test result can be delivered, for example using point-of-care testing (POCT), rather than sending a specimen to a central laboratory, can have a significant impact on the speed with which care can be delivered, the place in which it can be delivered (e.g. primary vs secondary care), as well as greater engagement with the patient. $\underline{13}$

3. A test may demonstrate a range of utilities, e.g. for screening, diagnosis, treatment selection and monitoring, as well as prognosis. This feature is particularly demanding on evidence of effectiveness, especially when considering personalised treatment, as well as in the development of a value proposition.14,15

4. The evidence base for diagnostic tests is also considered to be limited which may in part be due to the challenges in designing and performing evaluations. $\underline{16}$

5. Evidence indicates that a significant proportion of test results are not followed up..$\underline{17}$

6. There is the perception of a significant level of unnecessary testing, although more detailed analysis indicates that there is both inappropriate over- and under-testing. $\underline{.18}$

7. A wide variation in the use of specific investigations has been documented,,$\underline{19}$ although there is less knowledge of the actual level of 
testing that occurs within a pathway, as against the pathway prescribed in a clinical guideline. This situation may arise for a number of reasons, including poor adherence to a guideline, $\underline{20}$ inefficiencies in delivery, e.g. delay in obtaining results from investigations, other operational reasons, e.g. the impact of nonelective admissions to the intensive care unit, or departures from the guideline for clinical reasons, e.g. the occurrence of neutropenic sepsis in patients receiving chemotherapy. $\underline{\underline{21}}$

All these issues may arise because reimbursement of tests, as well as other elements of the care pathway, are managed on the basis of activity (inputs) rather than outcomes (outputs). $\underline{22}, \underline{23}$ Thus, in laboratory medicine investigations, reimbursement is based on the cost of the goods (technology) and the time to use the technology, i.e. produce the test result; this is often referred to as the "cost-per-test". Consequently, laboratory tests are often regarded as a commodity and operating within a silo, with little incentive to link the test result to any health outcome.

Donabedian advocated that quality in healthcare should be evaluated in terms of "structure", "process" and outcomes", which can be summarised as "who and what (the resources/"structure") are involved in delivering healthcare", "how (the process)" and "with what result" (the "outcome")..$\underline{24}$ This approach is relevant to both adoption of, and quality improvement in, the use of technology in healthcare delivery. It is implicit in Donabedian's consideration that there are a number of stakeholders involved in delivering healthcare which include patients, carers, purchasers 
of healthcare, providers of healthcare and their constituent elements. The latter are made up of inpatient facilities and staff, outpatient facilities and staff, diagnostic services all of which can be referred to as "service line providers". All stakeholders and service line providers will each have differing responsibilities and expectations in relation to the care pathway. In our consideration, we assume that purchasers, e.g. commissioners in the United Kingdom, to represent the broader perspective of patients and the wider population. Within the UK health economics perspective, the National Institute for Health and Clinical Excellence (NICE) and Healthcare Improvement Scotland (HIS), reference case for a technology appraisal

refers to the perspective on costs being NHS (National Health Service) and PSS (Personal social services), with outcomes based on all direct health effect for patients and when relevant, for carers as well. It does not recommend incorporating a broader societal perspective for evaluations such as the impact on patient or carer productivity losses nor impact on other sectors such as transport, justice, environment, etc.

We therefore propose that a clear link needs to be established between evidence of cost effectiveness, gained from the use of established tools of economic analysis, and resource allocation. It is only through this link that resource utilisation can be judged as cost effective.

\section{Limitations of current health economic tools informing adoption}

The core methodology of cost effectiveness analysis involves comparing the outcome expressed in terms of some measure of health gain, e.g. 
years of life, measure of mobility, clinical outcome, QALY, as the denominator, to the numerator or the cost associated with achieving the health gain. It has therefore been described as a means of comparing the costs of delivering an additional unit of health outcome. .25 It is one of a number of techniques employed in policymaking decisions, and has been applied to elements of the laboratory medicine services, e.g. use of cardiac markers in the diagnosis of acute coronary syndrome, $\underline{26}$ and use of natriuretic peptide measurement in the diagnosis of heart failure. $\underline{27}$

However, cost effectiveness analysis does not inform the detail of investment and disinvestment decisions required at the provider level when translating policymaking decisions into routine practice, e.g.using the resource, process and outcomes framework across the care pathway to deliver change whether it be adoption of a new test or quality improvement of current practice. This issue was highlighted in the NHS report Innovation, Health and Wealth which stated the need to "align financial, operational and performance incentives to support adoption and diffusion of innovation". 11

\section{Definition of translational health economics}

Translational health economics can be defined as a tool to enable translation of the findings from established tools of economic assessment in healthcare, such as cost effectiveness analysis and budget impact analysis (BIA), into routine clinical practice and providing guidance on resource utilisation in the delivery of healthcare, in order to obtain value-formoney for all stakeholders, and delivering an identifiable return on 
investment. It is therefore an important tool in the adoption of new technology in the delivery of healthcare.

\section{Tools for economic evaluation in the adoption of new tests}

A number of economic tools have been used when making the case for adopting a new test or testing device.

\section{Programme budgeting}

This technique, along with marginal analysis, is based on analysis of opportunity costs at the margin and is typically employed for priority setting when considering alternative opportunities including technologies. $\underline{28}$ It is most commonly used at the local healthcare purchasing level for care services $\underline{29}$ and focusses on a whole condition scenario, albeit encompassing different clinical settings, e.g. primary and secondary care, and is built around a population-based care pathway. It is not a technique with sufficient granularity to focus resource utilisation and the contribution of individual stakeholders in care pathways, e.g. the impact of laboratory medicine investigations on that pathway.

\section{BIA}

$\mathrm{BIA}$ is a tool that can provide more detailed costings for individual stakeholders and for the care pathway as a whole. These might be used to determine the full budget impact of an intervention once a decision using programme budgeting has been made to proceed. 
BIA measures the budget impact to the provider of a new intervention or modified care pathway over a one to five-year period. A driver of the need for BIA has been to overcome the problem of budget silos where the purchasers of technology, including laboratories, are rarely reimbursed from the cost benefits that accrue in other departments/service lines (silos). In many countries, organisations responsible for the assessment of new technology now insist on economic evidence beyond that of cost effectiveness analysis. BIA sets out to identify the utilisation, and cost, of the current and the new intervention. This will include the overall effect of the new intervention on what is called "the cost of illness" as well as the impact on condition related and indirect costs. $\underline{30}$ However, BIA does not have the detail to address the investment decisions related to the impact of a laboratory investigation for each of the stakeholder groups involved in delivering the care pathway, particularly when addressing quality improvement in relation to departures from a care pathway.

Several examples of BIA in relation to laboratory tests are described in the literature. These include assessment of the economic impact of POCT for $\mathrm{HbA1c}$ in the management of diabetes $\underline{31}$; use of a high sensitivity troponin assay to enable the use of a two-hour rule-out protocol for patients presenting with chest pain considered to be at low risk. $\underline{32}$

\section{Service line management}

The aim of service line management (SLM) is to improve the quality and productivity of services through the use of detailed information relating to performance and cost. The process was originally developed in the US in 
the 1980s, $\frac{33}{}$ and was adapted for the UK National Health Service by Monitor. It is a process that is usually applied to improve the management of an existing intervention through a bottom-up costing analysis. A key aspect of SLM is that it is a process intended for those responsible for managing the care process, namely clinicians, $\underline{33}$ and aimed, primarily at hospitals. In establishing the need for SLM, Monitor encouraged its use as part of the application process for becoming a Foundation Trust, but Monitor did not mandate its use and as the Kings Fund noted $\stackrel{33}{ }$ SLM is often an approach that struggles in a period of financial pressure. This has resulted in a rather limited adoption in the UK.

SLM can therefore be employed to establish the resource utilisation and costs of current practice, with that data then being used to inform expected resource utilisation in a revised pathway. Typical resource elements within a service line are illustrated in Table 1. This is an approach that offers greater detail of resource utilisation across a care pathway, or element of a care pathway, and can therefore be of value when considering the impact of a laboratory investigation in relation to process change and resource utilisation. In addition, a key element of SLM is to report income as well as cost and thereby highlighting issues of sustainability and value which will inform investment and disinvestment.

Table 1. The service lines and cost elements for a typical District General Hosp

\begin{tabular}{|l|l}
\hline Service lines & Cost elements \\
\hline Accident and emergency & Blood \\
\hline Acute medicine & CNST \\
\hline
\end{tabular}


Table 1. The service lines and cost elements for a typical District General Hosp

\begin{tabular}{|c|c|}
\hline Service lines & Cost elements \\
\hline Care of the elderly & Critical care \\
\hline Clinical support & Drugs \\
\hline Critical care & Emergency \\
\hline General surgery & Endoscopy \\
\hline Head and neck & High cost drugs \\
\hline Oncology & Imaging \\
\hline Paediatrics & Laboratory medicine \\
\hline Specialist medicine & Medical staff \\
\hline Trauma and orthopaedics & Non-clinical income \\
\hline \multirow[t]{13}{*}{ Women's services } & Non-patient care activities \\
\hline & Operating rooms \\
\hline & Other clinical and spec. nursing staff \\
\hline & Other clinical supplies and services \\
\hline & Other diagnostics \\
\hline & Other special procedure suites \\
\hline & Outpatients \\
\hline & Overheads \\
\hline & Pharmacy services \\
\hline & Prostheses/implants/devices \\
\hline & Radiotherapy \\
\hline & Therapies \\
\hline & Ward costs \\
\hline
\end{tabular}

The cost of a service line will comprise a combination of the relevant cost elements.

CNST: clinical negligence scheme for trusts.

\section{Time-driven activity-based costing}


This is an alternative finance-driven costing process that takes place in the audit or performance management phase of an existing intervention. It can also be used to inform a revised pathway although it might be necessary to initially apportion a cost to the new intervention, combined with sensitivity analysis, which can then be audited and revised during the course of implementation. It utilises a bottom-up approach to costing but it is more detailed than SLM since it relies on process mapping techniques to accurately measure all the human and technology resources that contribute to a particular process of care used to treat patients. The technique was developed in industry and is now being widely used in healthcare. $\underline{34}$ Examples include resource utilisation in outpatient clinics, $\underline{35}$ and process improvement in the Emergency Department. $\underline{36}$ The technique offers a more detailed analysis than SLM and identifies the resource utilisation at each step by each member of the provider team, and can be taken down to the individual patient level. $\underline{35}$ Thus, in quality improvement, it can identify which steps contribute to high costs, which steps might be made redundant or are not contributing to better outcomes and whether capacity, either equipment or human, is being used efficiently. This enables benchmarking across different sites providing the same care objectives or outcomes, a technique essential for achieving improved efficiencies. More generally TDABC is seen as a way to address problems such as the misalignment between reimbursement fees and delivery of services and to achieve the appropriate disinvestment of services which are often necessary after changes to the process of care that improve patient outcomes. 
The role of the tools outlined above, together with the place of translational health economics, are illustrated in the stages of technology innovation in Figure 1.

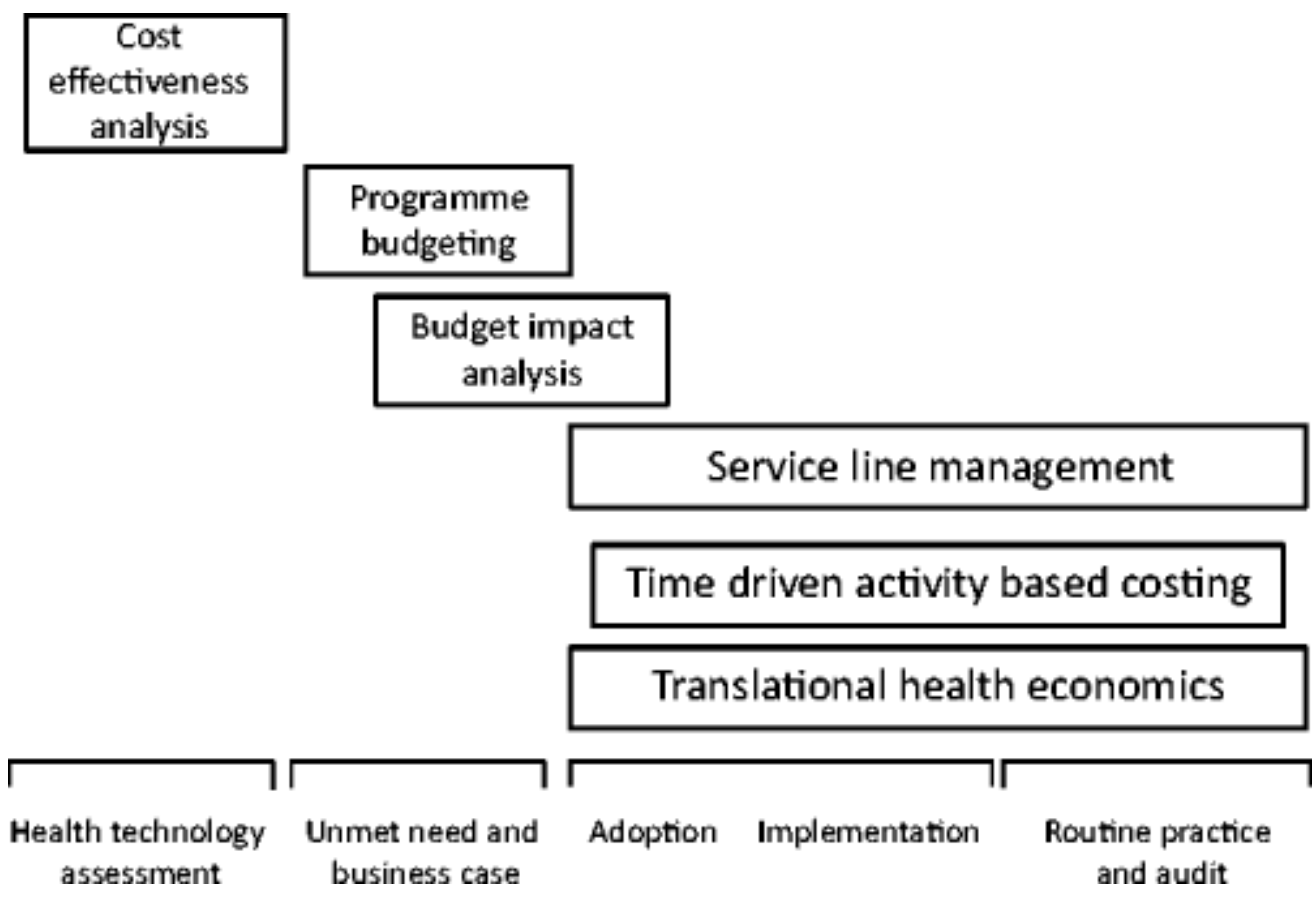

Figure 1. Map of the tools for economic assessment that can be used in the assessment and adoption of laboratory medicine interventions, including the place of translational health economics.

\section{Translational health economics explained}

The tools outlined above describe how forms of economic assessment can be employed across the spectrum of stakeholders involved in the organisation and delivery of healthcare. However, these techniques do not cover the spectrum of technology adoption from policymaking to practical service delivery at the provider level, with little guidance at the provider level that relate to resource utilisation. Thus, the application of these techniques does not fully address the issues raised at the outset of this 
discussion, particularly informing the practical issues of investment and disinvestment in routine practice at a provider level, limiting the capability to deliver the promised return on investment. We propose that these goals will only be met through the adoption of translational health economics.

The employment of translational health economics requires (i) evidence to demonstrate the clinical effectiveness of the intervention of interest, and that the intervention is relevant to the patient cohort and clinical setting in question, and (ii) evidence related to cost effectiveness of the intervention, including evidence from trials and economic modelling of cost effectiveness.

The core of translational health economics can be summarised in five steps:

Step 1. Defining the care pathway in which the intervention will be used, and the activities involved in delivering that pathway. The emphasis of this analysis is on "real world" experience, rather than a "theoretical pathway" protocol.

Step 2. Defining the resource utilisation in the current pathway, e.g. using TDABC and the costs involved, and the current funding or reimbursement made available to deliver the pathway, highlighting inefficiencies and adverse events including their costs and how they are reimbursed.

Step 3. Determine exactly where in the care pathway the new intervention (test) will be used, based on existing evidence. 
Step 4. Determine the changes in resource utilisation of the revised pathway, e.g. using TDABC, and the potential impact on all of the stakeholders that will result from introduction of the intervention.

Step 5. Assess the potential cost of the new pathway at the local level to identify the potential investment and disinvestment in resources. At this stage, it is also useful to identify potential measures for monitoring the pathway, e.g. time in hospital, number of clinic visits etc.

The information gained from translational health economics can then be used to develop both a value proposition and a business case for adoption of the new intervention and associated pathway, together with the detail of an implementation plan. Further these steps can be employed in both the development of new services, and the modification of existing services including quality improvement. This highlights the importance of designating staff to take responsibility for monitoring the adoption of new technology, as well as continuous quality improvement; the absence of the former has been recognised as a barrier to adoption of new technology. $\underline{8}$

The core of any project involving the use of translational health economics is (i) a map of the care pathway that outlines the process and the service lines involved in the delivery of the pathway, (ii) a set of metrics or outcome measures that enable adherence to the pathway to be assessed and the benefits to each of the stakeholders identified, and (iii) a clear identification of the resource investment (reimbursement/payment) and level of utilisation by each of the service lines involved in the delivery of care. This is illustrated in principle in Figure 2; the exact nature of reimbursement and 
service lines will vary according to the health system, as well as the individual pathways.
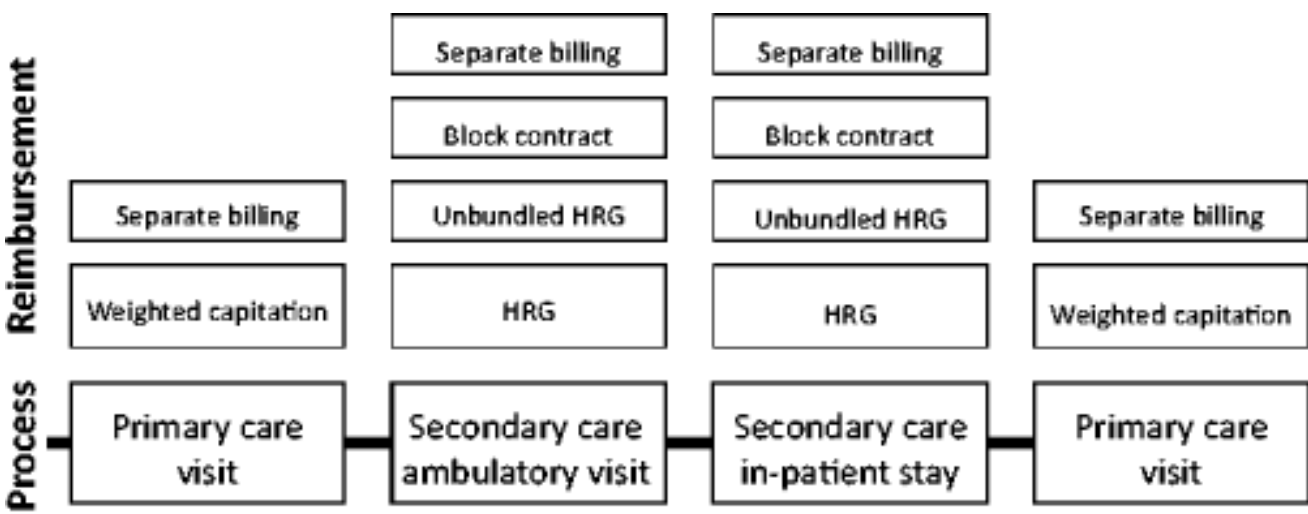

\section{Primary care visit}

1. Practice
2. Medical
2. Nursing
3.
4. Diagnostics
5. Drugs
5.
6. Administration
7. Overheads
을
1. Clinic
2. Medical
3. Nursing
4. Diagnostics
5. Drugs
1. Ward
2. Medical
3. Nursing
4. Diagnostics
5. Drugs \& bloods
6. Specialist therapy
7. Administration
8. Overheads
6. Emergency Dept
7. Intensive care
8. Operating Room
9. Specialist eqpt
10. Administration
11. Overheads

1. Practice

2. Medical

3. Nursing

4. Diagnostics

5. Drugs

6. Administration

7. Overheads

Figure 2. Illustration of the relationship between reimbursement and resource utilisation across a care pathway in the NHS, showing the different service lines that might be employed at different stages of the care pathway.

\section{Examples of the potential for use of translational economics}

The success of translational economics can be measured by the delivery of clinically effective care that addresses the needs of all stakeholders, and is associated with the optimal use of resources across the care pathway.

Below we describe three examples of the use of laboratory tests that enable a change in the care process where the steps of translational health economics, described earlier, can inform, and translate, the necessary investment and disinvestment decisions into resource allocation at the local 
practice level, which is required in order to achieve effective adoption and implementation.

In the first example, the BIA of POCT compared to laboratory testing for $\mathrm{HbA1C}$ in patients with diabetes, identified the main service line costs as being for the test (laboratory or POCT) and the physician visit; in this case using the reimbursement data to provide a measure of the costs of delivering the care pathway. $\underline{31}$ Thus, investment in POCT was more than paid for by reducing the number of ambulatory clinic visits (i.e. disinvestment) in those patients needing to improve their glycaemic control. In the second example, a high sensitivity troponin test is employed to facilitate earlier decision making in patients presenting to the Emergency Department with chest pain, enabling a two-hour rule-out protocol instead of a six-hour rule-out protocol. $\underline{32}$ The authors used a decision analytic costeffectiveness model based on the expected hospital cost and the length of stay in the Emergency Department to simulate the potential cost savings that accrue from investment in laboratory testing.

In the third example, a new test enabling earlier diagnosis of preeclampsia with improved sensitivity and specificity compared with current practice. A decision analytic model showed that while the new test increased the cost of the true positives, this was offset by a greater reduction in the cost of managing false positives and false negatives. $\frac{37}{3}$ The reduction in costs covers ambulatory clinic visits, hospital stay and treatment costs. 
In each of these BIA examples, the reimbursement data, or similar, have been used to identify the potential resource utilisation costs. However, this does not give the granularity of data at the service line level to inform the investment and disinvestment decisions associated with a change in pathway. We would argue that by employing the translational economic strategy indicated in the steps outlined about, it is possible to achieve more effective optimisation of resource utilisation. A simple way of viewing this is illustrated in Figure 3 which indicates where resource saving can be made.

\begin{tabular}{|l|}
\hline Ward \\
\hline \hline Clinic \\
\hline \hline Medical \\
\hline Nursing \\
\hline \hline Diagnostics \\
\hline \hline Drugs \& bloods \\
\hline \hline Emergency Dept \\
\hline \hline Intensive Care \\
\hline \hline Operating Room \\
\hline \hline Specialist eqpt \\
\hline \hline Administration \\
\hline \hline Overheads \\
\hline
\end{tabular}

(a)

(b)

(c)

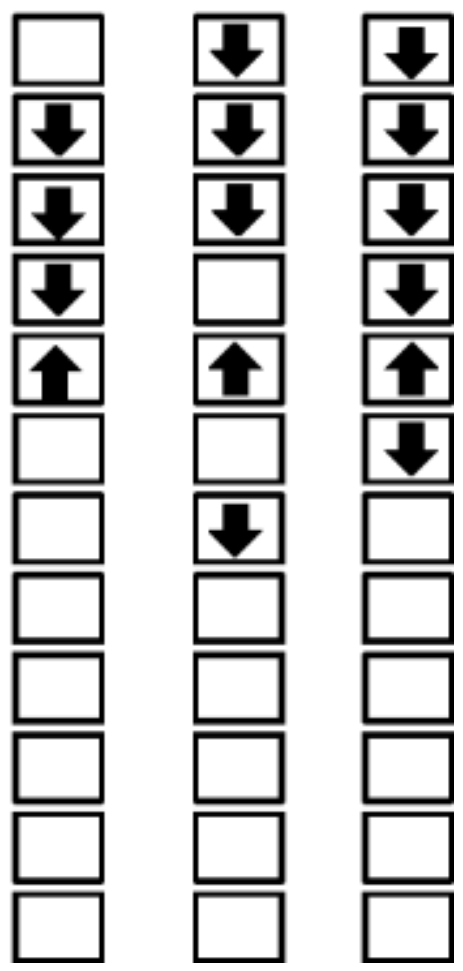

Figure 3. Summary charts for investment $(\uparrow)$ and disinvestment ( $\downarrow$ ) requirements in three scenarios for adoption of alternative diagnostic interventions, indicating the service lines most likely to be impacted: (a) the use of POCT for HbA1c in the management of patients with diabetes, (b) the introduction of a high sensitivity assay from troponin I in low-risk patients with chest pain presenting to the Emergency Department, (c) the introduction of a new test for the diagnosis of pre-eclampsia in pregnant women. 


\section{Discussion}

The intent of this paper is to show how various economic tools can be used to achieve value when employing laboratory medicine services in care pathways. We propose an approach that delivers the benefits and returnon-investment promised in clinical and cost effectiveness studies, through translation of these findings in to the potential changes expected in resource utilisation. An absolutely key requirement for the use of this methodology in practice is a better knowledge and understanding of how all the stakeholders involved in a care pathway could work together, both at an organisational and service line level.

The methodology we describe addresses the challenges of resource optimisation discussed by Henshall et al. $\underline{38}$ Importantly, it recognises that adoption of new technologies is best achieved at a local level, where there is likely to be a better understanding of current practice and the barriers to change. $\frac{39}{} \mathrm{~A}$ key part of such change is increased resources or investment for some areas (service lines) or stakeholders and reduction of resources or disinvestment for others. Getting the balance right between investment and disinvestment can be both difficult and unpopular, but the translational economic steps we have described play a key role in determination of the right balance. With the latter, there is a much better chance of getting an appropriate return on investment from new healthcare technology, the lack of which is a commonly described problem. $, \underline{1}, \underline{8}$

We acknowledge there are challenges to using the above approach, including: 
(i) There must be good data on the process of care and the associated resource utilisation required to deliver these processes, i.e. the product of service lines, (ii) investment in new technology is required at the outset of the adoption process, whether it be simply a new test or the requirement for a new piece of equipment, whereas the clinical outcomes may take a longer timeframe to deliver, (iii) disinvestment in large pieces of equipment or processes will take time to achieve, (iv) optimisation of processes may not release finite units of resource, e.g. a hospital bed, a member of staff, and (v) innovation and, even, quality improvement require change in practice.

We believe the above challenges are not insurmountable and with the appropriate information, together with a willingness by all stakeholders to work together, it should be possible using the economic tools we have described to translate population clinical and cost effectiveness evidence into efficient and effective local practice, providing value for money. Indeed, Lee $\underline{40}^{0}$ has suggested that a value-based approach to care is "likely to be the best opportunity of all stakeholders working together". We also believe that an adoption process informed by translational health economics will deliver the care pathway efficiency and productivity that is being sought. $\underline{1}, \underline{7}, \underline{11}$

\section{Acknowledgements}

The views expressed are those of the authors and not necessarily those of the NHS, the NIHR or the Department of Health. The study sponsors had 
no role in the design, analyses or reporting of the study. The researchers retained complete independence in the conduct of this study.

\section{Declaration of conflicting interests}

The author(s) declared no potential conflicts of interest with respect to the research, authorship, and/or publication of this article.

\section{Funding}

The author(s) disclosed receipt of the following financial support for the research, authorship, and/or publication of this article: This article presents independent research funded by the National Institute for Health Research (NIHR) Diagnostic Evidence Co-operative Oxford. $\underline{1}, \underline{2}$

\section{References}

1 Scotland G and Bryan S. Why do health economists promote technology adoption rather than the search for efficiency? A proposal for a change in our approach to economic evaluation in health care. Med Decis Making 2016, doi: 10.1177/0272989X16653397

2 Thompson M, Price C, Van den Bruel A, et al. Innovation in diagnostics and healthcare: improving bench to bedside processes for testing, www.oxford.dec.nihr.ac.uk/aboutus/innovation_in_diagnostics.pdf (accessed 31 July2017).

3 Institute of Medicine (Eds Yong PL, Saunders RS, and Olsen LA). The healthcare imperative. Lowering costs and improving outcomes. Workshop 
series summary, www.ncbi.nlm.nih.gov/books/NBK53920/ (accessed 31 July 2017).

4 Kaplan RS and Porter ME. How to solve the cost crisis in health care. Harv Bus Rev 2011; 89: 46-52. 5456-5461. passim.

5 Billings $\mathrm{J}$ and de Weger E. Contracting for integrated health and social care: a critical review of four models. J Integ Care 2015; 23: 153-175.

6 Mountford J and Davie C. Toward an outcomes-based health care system. A view from the United Kingdom. JAMA 2010; 304: 2407-2408.

7 Berwick DM and Hackbarth AD. Eliminating waste in US health care. JAMA 2012; 307: 1513-1516.

8 NHS Institute for Innovation and Improvement. Organisational and behavioural barriers to medical technology adoption, www.institute.nhs.uk/images/ResearchAndEvaluationReports/OB B_MTA.pdf (accessed 31 July 2017).

9 World Health Organization. Barriers to innovation in the field of medical devices. Background paper

6, http://whqlibdoc.who.int/hq/2010/WHO_HSS_EHT_DIM_10.6_eng.pdf. (2010, accessed 31 July 2017).

10 Robert G, Greenhalgh T, MacFarlane F, et al. Adopting and assimilating new non-pharmaceutical technologies into health care: a systematic review. J Health Serv Res Policy 2010; 15: 243-250. 
11 Department of Health. Innovation health and wealth: accelerating adoption and diffusion in the NHS, www.dh.gov.uk/health/2011/12/nhsadoptinginnovation/ (accessed 31 July 2017).

12 Craig P, Dieppe P, Macintyre S, et al. Developing and evaluating complex interventions: new guidance. Medical research council. BMJ 2008; 337: a1655

13 St Sohn A and Price CP. Economic evidence and point-ofcare testing. Clin Biochem Rev 2013; 34: 61-64.

14 Price CP and St John A. Anatomy of a value proposition for laboratory medicine. Clin Chim Acta 2014; 436C: 104-111.

15 Price CP, John AS, Christenson R, et al. Leveraging the real value of laboratory medicine with the value proposition. Clin Chim Acta 2016; 462: 183-186.

16 Price CP. Evidence-based laboratory medicine: is it working in practice? Clin Biochem Rev 2012; 33: 13-19.

17 Callen J, Georgiou A, Li J, et al. The safety implications of missed test results for hospitalised patients: a systematic review. BMJ Qual Saf 2011; 20: 194-199.

18 Zhi M, Ding EL, Theisen-Toupal J, et al. The landscape of inappropriate laboratory testing: a 15-year meta-analysis. PLoS One. 2013; 8: e78962. doi:10.1371/journal.pone.0078962 
19 Right Care. The NHS atlas of variation in diagnostic services, www.rightcare.nhs.uk/downloads/Right_Care_Diagnostics_Atlas hi-res.pdf (accessed 31 July 2017).

20 Driskell OJ, Holland D, Hanna FW, et al. Inappropriate requesting of glycated hemoglobin (HbA1c) is widespread: assessment of prevalence, impact of national guidance, and practice-to-practice variability. Clin Chem 2012; 58: 906-915.

21 Baker K, Dunwoodie E, Jones RG, et al. Process mining routinely collected electronic health records to define real-life clinical pathways during chemotherapy. Int J Med Inform 2017; 103: 32-41.

22 St John A, Edwards G, Fisher S, et al. A call for a value based approach to laboratory medicine funding. Clin Biochem 2015; 48: 823-826.

23 Erstad BL. Value-based medicine: dollars and sense. Crit Care Med 2016; 44: 375-380.

24 Donabedian A. An introduction to quality assurance in healthcare. Oxford: Oxford University Press, 2003, p.200.

25 The Kings Fund. The NHS productivity challenge: experience from the frontline, www.kingsfund.org.uk/sites/files/kf/field/field_publication_file/thenhs-productivity-challenge-kingsfund-may14.pdf (accessed 31 July 2017).

26 Goodacre S, Thokala P, Carroll C, et al. Systematic review, metaanalysis and economic modelling of diagnostic strategies for suspected acute coronary syndrome. Health Technol Assess 2013; 17: 1 
27 Mueller C. Cost-effectiveness of B-type natriuretic peptide testing. Congest Heart Fail 2008; 14: 35-37.

28 Goodwin E and Frew EJ. Using programme budgeting and marginal analysis (PBMA) to set priorities: reflections from a qualitative assessment in an English Primary Care Trust. Soc Sci Med 2013; 98: 162-168.

29 Charles JM, Brown G, Thomas K, et al. Use of programme budgeting and marginal analysis as a framework for resource reallocation in respiratory care in North Wales, UK. J Public Health 2015; Sep 16. pii: fdv12.

30 Sullivan SD, Mauskopf JA, Augustovski F, et al. Budget impact analysisprinciples of good practice: report of the ISPOR 2012 budget impact analysis good practice II task force. Value Health 2014; 17: 5-14.

31 Chadee A, Blackhouse G and Goeree R. Point-of-care hemoglobin A1c testing: a budget impact analysis. Ontario Health Technol Assess Ser 2014; 14: 1-23.

32 Cheng Q, Greenslade JH, Parsonage WA, et al. Change to costs and lengths of stay in the emergency department and the Brisbane protocol: an observational study. BMJ Open 2016; 6: e009746 doi:10.1136/bmjopen2015-009746.

33 The Kings Fund. Service-line management Can it improve quality and efficiency? www.kingsfund.org.uk/sites/files/kf/service-linemanagementquality-efficiency-kings-fund-january2011.pdf (accessed 31 July 2017). 
34 Kaplan RS, Witkowski M, Abbott M, et al. Using time driven activitybased costing to identify value-improvement opportunities in health care. $J$ Health Manag 2014; 59: 399-413.

35 Waago-Hansen C. How time-driven activity-based costing (TDABC) enables better use of existing resources in order to improve return on investment (ROI) in modern healthcare and hence facilitates a sustainable healthcare system. theHealth 2014; 5: 3-8.

36 Yun BJ, Prabhakar AM, Warsh J, et al. Time-driven activitybased costing in emergency medicine. Ann Emerg Med 2016; 67: 765-772.

37 Hadker N, Garg S, Costanzo C, et al. Financial impact of a novel preeclampsia diagnostic test versus standard practice: a decisionanalytic modeling analysis from a UK healthcare payer perspective. $\mathrm{J}$ Med Econ 2010; 13: 728-737.

38 Henshall C, Schuller T and Mardhani-Bayne L. Using health technology assessment to support optimal use of technologies in current practice: the challenge of "disinvestment". Int J Technol Assess Health

Care 2012; 28: 203-210.

39 Kyratsis $\mathrm{Y}$, Ahmad R and Holmes A. Technology adoption and implementation in organisations: comparative case studies of 12 English NHS Trusts. BMJ Open 2012; 2: e000872 doi:10.1136/bmjopen-2012000872. 
40 Lee TH. Putting the value framework to work. N Engl J

Med 2010; 363: 2481-2483. 